\title{
Holographic Formulation of Quantum Supergravity
}

\author{
Yi Ling ${ }^{\dagger}$ and Lee Smolin** \\ Center for Gravitational Physics and Geometry \\ Department of Physics \\ The Pennsylvania State University \\ University Park, PA, USA 16802 \\ and \\ The Blackett Laboratory \\ Imperial College of Science, Technology and Medicine \\ South Kensington, London SW7 2BZ, UK
}

October 24, 2018

\begin{abstract}
We show that $\mathcal{N}=1$ supergravity with a cosmological constant can be expressed as constrained topological field theory based on the supergroup $O s p(1 \mid 4)$. The theory is then extended to include timelike boundaries with finite spatial area. Consistent boundary conditions are found which induce a boundary theory based on a supersymmetric Chern-Simons theory. The boundary state space is constructed from states of the boundary supersymmetric Chern-Simons theory on the punctured two sphere and naturally satisfies the Bekenstein bound, where area is measured by the area operator of quantum supergravity.
\end{abstract}

*email:†ling@phys.psu.edu ,* smolin@phys.psu.edu 


\section{Contents}

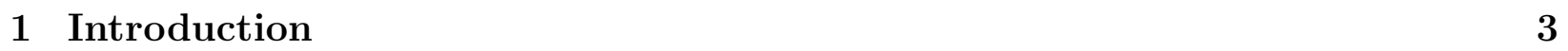

\begin{tabular}{|lll}
\hline 2 & Supergravity as a constrained topological field theory & 6
\end{tabular}

3 The Canonical Formalism of $\mathrm{N}=1$ SCBF theory 9

3.1 The $3+1$ decomposition of the action. . . . . . . . . . . . . . . . 10

3.2 The primary constraints . . . . . . . . . . . . . . . . . . . 11

3.3 Treatment of the fermionic constraints: method 1: . . . . . . . . . . . . . . 13

3.4 Treatment of the fermionic constraints: method 2: . . . . . . . . . . . . . 13

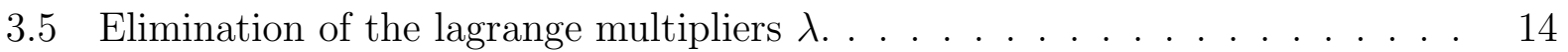

3.6 The Gauss and supersymmetry constraints . . . . . . . . . . . . . . . . . . . 15

3.7 The hamiltonian and diffeomorphism Constraints . . . . . . . . . . . . . . . 17

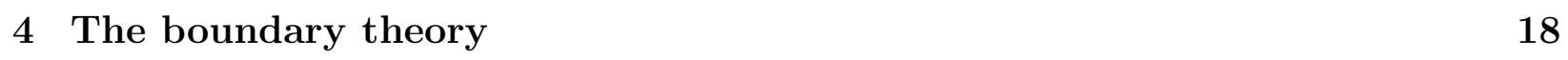

4.1 Boundary terms in the action and equations of motion . . . . . . . . . . . . 18

4.2 Boundary terms in the canonical theory . . . . . . . . . . . . . . . . . . 21

5 Quantization of the theory $\quad 23$

\begin{tabular}{lll}
\hline 6 Conclusions & 25
\end{tabular} 


\section{Introduction}

In this paper we describe a formulation of quantum supergravity with a finite cosmological constant, in the presence of a timelike boundary with finite spatial area. We show that, as in the case of quantum general relativity [1, 2], a holographic formulation of the theory naturally emerges. This work is part of a series of papers [3, 4, 5] in which we are extending to quantum supergravity the methods [6, 6, 8, 9, 10] which have been developed successfully to formulate quantum general relativity円.

The particular interest in this paper is the form of the boundary theory, which turns out to be built from the states of a supersymmetric Chern-Simons theory based on the superalgebra $O s p(1 \mid 2) \oplus O s p(1 \mid 2)$. We believe that, when extended to the $\mathcal{N}=8$ case, these results will be significant for the understanding of the AdS/CFT conjecture in $3+1$ dimensions. The extension of our results to the $N=2$ case may also make possible the detailed comparisons of the string theory and loop quantum gravity description of boundaries and horizons[四]. These questions will be the subject of further papers in this series.

Along the way, the key idea that we exploit is that supergravity, as well as general relativity [1], can be understood as a constrained topological quantum field theory. This emerged already in several papers [13, 14]. This fact is responsible for the characteristic form of the boundary theory, as made up of conformal blocks coming from representations of topological field theory.

For the case in which spacetime has a time like boundary with finite area, the holographic principle can be stated as follows [1, 2, 15, 16, 17, 18.

1) The hilbert space $\mathcal{H}_{\mathcal{B}}$ for all states of a quantum gravity theory on the interior of a spacial manifold $\mathcal{M}$ must be decomposable in terms of eigenspaces of an observable $\hat{A}$ that measures the area of the boundary $\mathcal{B}=\partial \mathcal{M}$. Thus,

$$
\mathcal{H}_{\mathcal{B}}=\sum_{a} \mathcal{H}_{a}
$$

where $a$ are the eigenvalues of the area operator $\hat{A}$. We write the decomposition as a sum because $\hat{A}$ is has been shown to have a discrete spectra, assuming only that it may be regulated in a way that results in an operator that is finite and diffeomorphism invariant [6].

\footnotetext{
${ }^{1}$ Earlier papers in this direction include 11, 12, 13, 14.
} 
2) The physical state spaces $\mathcal{H}_{a}$ must have finite dimension bounded by

$$
\operatorname{dim}\left(\mathcal{H}_{A}\right) \leq e^{\frac{a}{4 G \hbar}} .
$$

3) A complete measurement of a state in $\mathcal{H}_{\mathcal{B}}$ must be possible using only elements of an observable algebra $\mathcal{A}_{\mathcal{B}}$ associated with measurements that can be made on the boundary $\mathcal{B}$. The area $\hat{A}$ must be one of these observables.

This is a powerful clue, because quantum field theories with finite dimensional Hilbert spaces are not common (even the harmonic oscillator has an infinite dimensional Hilbert space.) The only large class of such theories are topological quantum field theories (TQFT). A natural strategy for formulating holographic quantum theories of gravity is then to look for quantum field theories in $d+1$ dimensions that induce TQFT's on their $d$ dimensional boundaries. One advantage of such a formulation is that it is already in a language which is background independent and non-perturbative.

As shown in [1, 2], quantum general relativity is exactly this kind of theory, as it can be understood to arise from a topological field theory by the imposition of certain constraints. We have also found this to be true of 11 dimensional supergravity, at least at the classical level[28]. The main goal of this paper is to extend that analysis to quantum supergravity.

In all these cases there is a set of exact physical quantum states in the bulk, associated with the boundary states [19, 20]. Besides being exact descriptions of the Planck scale structure these states also have semiclassical interpretations in terms of fluctuations around AntideSitter or deSitter spacetimes [19, 21].

That such results are possible at all may seem very mysterious, given that these theories are perturbatively non-renormalizable. They are possible because these are not just any non-renormalizable theories; they have special structures, which are in fact closely related to topological field theories. In fact, general relativity and supergravity ( at least for $N=1,2$ ) can be formulated as constrained topological field theories. This means that the derivative and boundary terms in the action are the same as in a related topological quantum field theory. The local degrees of freedom are introduced by a set of local constraints that do not involve the derivatives of the gravitational fields, and so do not alter the commutation relations of the quantum theory. It is this special structure that makes possible the holographic formulation of these theories, as well as a long list of other non-perturbative results, in both the canonical 
22] and path-integral [23, 24] frameworks.

A holographic formulation has been constructed for the quantum general relativity.In fact, it has been known for some time that general relativity in $3+1$ dimensions with a non-vanishing cosmological constant can be formulated in such a way that it induces a $2+1$ dimensional TQFT on its finite boundary. This theory has exactly the form just described, where the finite dimensional Hilbert spaces on the finite boundaries of a given area are built from the conformal blocks of an $S U(2)_{q}, W Z W$ conformal field theories on punctured spheres [1]. The area of the boundary is given by the sum of the total spins of the punctures, and the level $k$ is related to the cosmological constant by $k=6 \pi / G^{2} \Lambda$ [1, 25]. This new formulation is treated in [1]. The basic framework developed there is based on a representation of general relativity as a constrained topological field theory $(T F T)$. The starting point is a $T F T$ for an algebra $G$, taken to be $S p(4)$. This theory has no local degrees of freedom, but induces degrees of freedom on finite boundaries which arise from a $2+1$ dimensional Chern-Simons theory on the boundary. The local degrees of freedom are introduced by imposing constraints, which break the gauge symmetry to a subgroup $H=S U(2)_{L} \oplus S U(2)_{R}$. The result is that the physical degrees of freedom live in the coset $G / H$. The degrees of freedom on the boundary are also restricted to those of a Chern-Simons theory for $H$, but with curvatures constrained by the degrees of freedom in the coset, which turn out to parameterize the induced metric in the boundary. Further, the generators of the gauge transformations for the coset $G / H$ do not disappear, they instead emerge as the generators of four dimensional diffeomorphisms. Extended to the boundary theory, they define the Hamiltonian of the theory.

In this paper we extend this construction to the $\mathrm{N}=1$ chiral supergravity. We organize the paper as follows. In section two we rewrite the $\mathrm{N}=1$ chiral supergravity 11 in the formulation of $O \operatorname{sp}(1 \mid 4)$ constrained super $B F$ theory. Then in the following section we give the canonical formalism of the theory. The boundary formulation of the model which has finite boundary is described in section four. In section five we give the quantum mechanical description of the theory, and show that the space of boundary states can be constructed from the state space of an $O s p(1 \mid 2) \oplus O s p(1 \mid 2)$ Chern-Simons theory. We show that as in quantum general relativity, the Bekenstein bound is naturally satisfied[1]. We conclude the paper with a discussion of future directions. 


\section{Supergravity as a constrained topological field the- ory}

$\mathcal{N}=1$ supergravity can be written in a chiral formulation in terms of the pure spin connection [11. Here we'll show it can be written down directly as a constrained $B \wedge F$ theory by extending the algebra of the connection from $S p(4)$ to $O s p(1 \mid 4, R)$. This is similar to the way that supergravity was established as a constrained BF theory by Sano [13] and Ezawa [14.

The $O s p(1 \mid 4)$ superalgebra is a graded extension of the $\mathrm{Sp}(4)$ Lie algebra. It is generated by bosonic generators $J_{A B}, J_{A^{\prime} B^{\prime}}$, and $J_{A A^{\prime}}$ which span the $\operatorname{sp}(4)$ algebra and fermionic generators $Q_{A}$ and $Q_{A^{\prime}}$, where $\left(A, A^{\prime}\right)$ is a pair of $S U(2)$ indices corresponding to $S U(2) \oplus S U(2) \subset$ $S p(4)$. The superalgebra is

$$
\begin{aligned}
& {\left[J_{A B}, J^{C D}\right]=\delta_{(A}^{(C} J_{B)}^{D)}, \quad\left[J_{A^{\prime} B^{\prime}}, J^{C^{\prime} D^{\prime}}\right]=\delta_{\left(A^{\prime}\right.}^{\left(C^{\prime}\right.} J_{\left.B^{\prime}\right)}^{\left.D^{\prime}\right)},} \\
& {\left[J_{A A^{\prime}}, J_{B B^{\prime}}\right]=\epsilon_{A B} J_{A^{\prime} B^{\prime}}+\epsilon_{A^{\prime} B^{\prime}} J_{A B}, \quad\left\{Q_{A}, Q_{B}\right\}=\frac{G \sqrt{\Lambda}}{2} J_{A B},} \\
& \left\{Q_{A^{\prime}}, Q_{B^{\prime}}\right\}=\frac{G \sqrt{\Lambda}}{2} J_{A^{\prime} B^{\prime}}, \quad\left\{Q_{A}, Q_{A^{\prime}}\right\}=\frac{G \sqrt{\Lambda}}{2} J_{A A^{\prime}}, \\
& {\left[J_{A B}, Q_{C}\right]=\epsilon_{C(A} Q_{B)}, \quad\left[J_{A^{\prime} B^{\prime}}, Q_{C^{\prime}}\right]=\epsilon_{C^{\prime}\left(A^{\prime}\right.} Q_{\left.B^{\prime}\right)},} \\
& {\left[J_{A A^{\prime}}, Q_{B}\right]=\epsilon_{A B} Q_{A^{\prime}}, \quad\left[J_{A A^{\prime}}, Q_{B^{\prime}}\right]=\epsilon_{A^{\prime} B^{\prime}} Q_{A},} \\
& {\left[J_{A B}, Q_{C^{\prime}}\right]=0, \quad\left[J_{A^{\prime} B^{\prime}}, Q_{C}\right]=0,}
\end{aligned}
$$

where $G$ is the bare gravitational constant and the $\Lambda$ is cosmology constant.

The supertrace, Str, may be defined acting on the generators. The non-vanishing terms are

$$
\begin{aligned}
& \operatorname{STr}\left\{J_{A B} J^{C D}\right\}:=\delta_{(A}^{(C} \delta_{B)}^{D)} \quad \operatorname{STr}\left\{J_{A^{\prime} B^{\prime}} J^{C^{\prime} D^{\prime}}\right\}:=\delta_{\left(A^{\prime}\right.}^{\left(C^{\prime}\right.} \delta_{\left.B^{\prime}\right)}^{\left.D^{\prime}\right)} \quad \operatorname{STr}\left\{J_{A A^{\prime}} J^{B B^{\prime}}\right\}:=\delta_{A}^{B} \delta_{A^{\prime}}^{B^{\prime}} \\
& \operatorname{STr}\left\{Q_{A} Q_{B}\right\}:=\frac{1}{\mu} \epsilon_{A B} \quad \operatorname{STr}\left\{Q_{A^{\prime}} Q_{B^{\prime}}\right\}:=\frac{1}{\mu} \epsilon_{A^{\prime} B^{\prime}} .
\end{aligned}
$$

The configuration variables of the theories we will study here are the components of the connection one form of $O s p(1 \mid 4)$.

$$
\mathcal{A}=A^{A B} J_{A B}+A^{A^{\prime} B^{\prime}} J_{A^{\prime} B^{\prime}}+\frac{1}{l} e^{A A^{\prime}} J_{A A^{\prime}}+\psi^{A} Q_{A}+\chi^{A^{\prime}} Q_{A^{\prime}},
$$


where $l$ is a constant with the dimension of length. We see that it includes the vierbein one-form $e^{A A^{\prime}}$ and gravitino and anti-gravitino one forms $\psi^{A}$ and $\chi^{A^{\prime}}$.

The curvature two form $\mathcal{F}$ is

$$
\begin{aligned}
\mathcal{F}: & =d \mathcal{A}+[\mathcal{A}, \mathcal{A}] \\
& =F_{A B} J^{A B}+F_{A^{\prime} B^{\prime}} J^{A^{\prime} B^{\prime}}+F_{A A^{\prime}} J^{A A^{\prime}}+F_{A} Q^{A}+F_{A^{\prime}} Q^{A^{\prime}}
\end{aligned}
$$

The components of the curvature are,

$$
\begin{gathered}
F_{A B}=f_{A B}+\frac{1}{l^{2}} e_{A A^{\prime}} \wedge e_{B}^{A^{\prime}}+\frac{G \sqrt{\Lambda}}{2 l} \psi_{A} \wedge \psi_{B}, \\
F_{A}=d \psi_{A}+A_{A}^{B} \wedge \psi_{B}-\frac{1}{l} e_{A A^{\prime}} \wedge \chi^{A^{\prime}}=D \psi_{A}-\frac{1}{l} e_{A A^{\prime}} \wedge \chi^{A^{\prime}} \equiv f_{A}-\frac{1}{l} e_{A A^{\prime}} \wedge \chi^{A^{\prime}}, \\
F_{A^{\prime} B^{\prime}}=f_{A^{\prime} B^{\prime}}+\frac{1}{l^{2}} e_{A^{\prime} A} \wedge e_{B^{\prime}}^{A}+\frac{G \sqrt{\Lambda}}{2 l} \chi_{A^{\prime}} \wedge \chi_{B^{\prime}}, \\
F_{A^{\prime}}=d \chi_{A^{\prime}}+A_{A^{\prime}}^{B^{\prime}} \wedge \chi_{B^{\prime}}-\frac{1}{l} e_{A^{\prime} A} \wedge \psi^{A}=\bar{D} \chi_{A^{\prime}}-\frac{1}{l} e_{A^{\prime} A} \wedge \psi^{A} \equiv f_{A^{\prime}}-\frac{1}{l} e_{A^{\prime} A} \wedge \psi^{A}, \\
F_{A A^{\prime}}=d e_{A A^{\prime}}+A_{A}^{B} \wedge e_{B A^{\prime}}+A_{A^{\prime}}^{B^{\prime}} \wedge e_{A B^{\prime}}+\frac{G \sqrt{\Lambda}}{2 l} \psi_{A} \wedge \chi_{A^{\prime}} .
\end{gathered}
$$

To construct the action we will need a Lie algebra valued two form $\mathcal{B}$, whose components are labeled.

$$
\mathcal{B}:=B_{A B} J^{A B}+B_{A^{\prime} B^{\prime}} J^{A^{\prime} B^{\prime}}+B_{A A^{\prime}} J^{A A^{\prime}}+\mu B_{A} Q^{A}+\mu B_{A^{\prime}} Q^{A^{\prime}}
$$

We can now write the action for the $O s p(1 \mid 4) B F$ theory.

$$
-i \mathcal{I}=-i \int_{M} \operatorname{STr}\left\{\frac{1}{g^{2}} \mathcal{B} \wedge \mathcal{F}-\frac{e^{2}}{2} \mathcal{B} \wedge \mathcal{B}\right\}
$$

Here $g, e$ are dimensionless constants. Note that $\mu$ appearing in (4) and (12) is another dimensionless constant.

The super- $B F$ theory is a topological quantum field theory in that it has no local degrees of freedom We now follow the method introduced in [2] and construct the action for supergravity by constraining the fields of the topological super- $B F$ theory. To do this we break some of the gauge invariance by imposing local, non-derivative constraints. The supergravity action is

$$
-i \mathcal{I}^{\text {initial }}=-i \mathcal{I}^{S B F}-i \mathcal{I}^{\text {Constraint }}
$$


where

$$
I^{\text {Constraint }}=\int_{\mathcal{M}} \lambda^{A B}\left(\frac{1}{l^{2}} e_{A A^{\prime}} \wedge e_{B} A^{\prime}-B_{A B}\right)+\lambda^{A}\left(\frac{1}{l} e_{A A^{\prime}} \wedge \chi^{A^{\prime}}-B_{A}\right) .
$$

In components this action is

$$
\begin{aligned}
-i \mathcal{I}^{\text {initial }}= & \int_{\mathcal{M}} \frac{1}{g^{2}}\left(B_{A B} \wedge F^{A B}+\frac{1}{l} B_{A} \wedge F^{A}\right)-\frac{e^{2}}{2}\left(B_{A B} \wedge B^{A B}+\frac{\mu}{l} B_{A} \wedge B^{A}\right) \\
& +\frac{1}{g^{2}}\left(B_{A^{\prime} B^{\prime}} \wedge F^{A^{\prime} B^{\prime}}+\frac{1}{l} B_{A^{\prime}} \wedge F^{A^{\prime}}\right)-\frac{e^{2}}{2}\left(B_{A^{\prime} B^{\prime}} \wedge B^{A^{\prime} B^{\prime}}+\frac{\mu}{l} B_{A^{\prime}} \wedge B^{A^{\prime}}\right) \\
& +\frac{1}{g^{2}}\left(B_{A A^{\prime}} \wedge F^{A A^{\prime}}-\frac{e^{2}}{2} B^{A A^{\prime}} \wedge B_{A A^{\prime}}\right) \\
& +\lambda^{A B}\left(\frac{1}{l^{2}} e_{A A^{\prime}} \wedge e_{B}^{A^{\prime}}-B_{A B}\right)+\lambda^{A}\left(\frac{1}{l} e_{A A^{\prime}} \wedge \chi^{A^{\prime}}-B_{A}\right) \\
& +\lambda^{A^{\prime} B^{\prime}}\left(\frac{1}{l^{2}} e_{A A^{\prime}} \wedge e_{B}^{\prime}{ }^{A}-B_{A^{\prime} B^{\prime}}\right)+\lambda^{A^{\prime}}\left(\frac{1}{l} e_{A A^{\prime}} \wedge \psi^{A}-B_{A^{\prime}}\right) .
\end{aligned}
$$

Note that the field equation for $B^{A A^{\prime}}$ yields,

$$
F_{A A^{\prime}}=B_{A A^{\prime}}
$$

Thus $B_{A A^{\prime}}$ carries the information as to the torsion, so that

$$
B_{A A^{\prime}}=\nabla e_{A A^{\prime}}+\frac{G \sqrt{\Lambda}}{2 l} \psi_{A} \wedge \chi_{A^{\prime}}
$$

The other field equations will eventually set $B^{A A^{\prime}}$ to zero, hence this part of the action is redundant and can be dropped. This simplifies the resulting canonical analysis, but does not affect the field equations. Once this is done the action splits into left and right handed pieces

$$
-i \mathcal{I}^{S G}=-i \mathcal{I}_{L}^{S G}+-i \mathcal{I}_{R}^{S G}
$$

where

$$
\begin{aligned}
-i \mathcal{I}_{L}^{S G}= & \int_{\mathcal{M}} \frac{1}{g^{2}}\left(B_{A B} \wedge F^{A B}+\frac{1}{l} B_{A} \wedge F^{A}\right)-\frac{e^{2}}{2}\left(B_{A B} \wedge B^{A B}+\frac{\mu^{2}}{l} B_{A} \wedge B^{A}\right) \\
& +\lambda^{A B}\left(\frac{1}{l^{2}} e_{A A^{\prime}} \wedge e_{B}^{A^{\prime}}-B_{A B}\right)+\lambda^{A}\left(\frac{1}{l} e_{A A^{\prime}} \wedge \chi^{A^{\prime}}-B_{A}\right) .
\end{aligned}
$$

The right handed piece is formally the conjugate of the left handed piece that results from switching primed and unprimed indices. We will see shortly how the reality conditions arise, whose effect will be to require that they are complex conjugates of each other.

To see that (20) is an action for $N=1$ supergravity we proceed to solve the constraint for $B_{A B}$ and $B_{A}$ 


$$
\begin{aligned}
B_{A B} & =\frac{1}{l^{2}} e_{A A^{\prime}} \wedge e_{B}{ }^{A^{\prime}}, \\
B_{A} & =\frac{1}{l} e_{A A^{\prime}} \wedge \chi^{A^{\prime}} .
\end{aligned}
$$

Substituting the solutions to (20), we find the action taking the form

$$
\begin{aligned}
-i \mathcal{I}_{L}= & \int_{\mathcal{M}} \frac{1}{g^{2} l^{2}}\left(e_{A A^{\prime}} \wedge e_{B} A^{\prime} \wedge f^{A B}+e_{A A^{\prime}} \wedge \chi^{A^{\prime}} \wedge D \psi^{A}\right) \\
& -\frac{1}{l^{4}}\left(\frac{1}{g^{2}}-\frac{e^{2}}{2}\right)\left(e_{A A^{\prime}} \wedge e_{B}^{A^{\prime}} \wedge e^{A B^{\prime}} \wedge e^{B}{ }_{B^{\prime}}\right)+\frac{\sqrt{\Lambda}}{2 l}\left(e_{A A^{\prime}} \wedge e_{B}{ }^{A^{\prime}} \wedge \psi^{A} \wedge \psi^{B}\right) \\
& -\frac{1}{l}\left(\frac{e^{2} \mu}{2 l^{2}}+\frac{1}{g^{2} l^{2}}\right)\left(e_{A A^{\prime}} \wedge \chi^{A^{\prime}} \wedge e^{A}{ }_{B^{\prime}} \wedge \chi^{B^{\prime}}\right) .
\end{aligned}
$$

If we define the dimensionless constants as follows

$$
G:=g^{2} l^{2} ; \quad \Lambda:=\frac{6}{l^{4}}\left(\frac{1}{g^{2}}-\frac{e^{2}}{2}\right) ; \quad \mu:=\frac{G \sqrt{\Lambda}-6}{3 g^{2} e^{2}}
$$

Then:

$$
\begin{aligned}
-i \mathcal{I}_{L}= & \int_{\mathcal{M}} \frac{1}{G}\left(e_{A A^{\prime}} \wedge e_{B}^{A^{\prime}} \wedge f^{A B}+e_{A A^{\prime}} \wedge \chi^{A^{\prime}} \wedge D \psi^{A}\right) \\
& -\frac{\Lambda}{6}\left(e_{A A^{\prime}} \wedge e_{B}^{A^{\prime}} \wedge e^{A B^{\prime}} \wedge e_{B^{\prime}}^{B}\right) \\
& +\frac{\sqrt{\Lambda}}{2 l}\left(e_{A A^{\prime}} \wedge e_{B}^{A^{\prime}} \wedge \psi^{A} \wedge \psi^{B}\right)-\frac{\sqrt{\Lambda}}{6 l}\left(e_{A A^{\prime}} \wedge \chi^{A^{\prime}} \wedge e_{B^{\prime}}^{A} \wedge \chi^{B^{\prime}}\right) .
\end{aligned}
$$

This is the same action as the CDJ formalism [26] after we solve the constraint equations associated to the Lagrangian multipliers $\phi_{A B C D}$ and $\kappa_{A B C}$ [11. We may note that the cosmological constant is zero if $e^{2} g^{2}=2$, at which point $\mu=-1$.

\section{The Canonical Formalism of $\mathrm{N}=1 \mathrm{SCBF}$ theory}

We now study the canonical formalism for $N=1$ supergravity based on the fields just introduced. Our main goal is to understand those issues which arise in the supergravity case. These mainly have to do with how the anticommutation relations between the left and right handed supersymmetry constraints arise and how they come to close on the spatial diffeomorphism and hamiltonian constraints.

In this section we ignore the possibility of boundary terms. Many of the expressions will later be modified by the presence of boundary terms. 


\subsection{The $3+1$ decomposition of the action.}

In the last section we see that $N=1$ supergravity can be written as a constrained topological field theory based on the supergroup $O s p(1 \mid 4)$. The total action can be expressed in terms of the component fields

$$
\begin{aligned}
-i \mathcal{I}= & -i \int_{M} d x^{4}\left\{\frac{1}{g^{2}}\left(B_{A B} \wedge F^{A B}+\frac{1}{l} B_{A} \wedge F^{A}\right)-\frac{e^{2}}{2}\left(B_{A B} \wedge B^{A B}+\frac{\mu^{2}}{l} B_{A} \wedge B^{A}\right)\right. \\
& +\lambda^{A B}\left(\frac{1}{l^{2}} e_{A A^{\prime}} \wedge e_{B}^{A^{\prime}}-B_{A B}\right)+\lambda^{A}\left(\frac{1}{l} e_{A A^{\prime}} \wedge \chi^{A^{\prime}}-B_{A}\right) \\
& -\frac{1}{g^{2}}\left(B_{A^{\prime} B^{\prime}} \wedge F^{A^{\prime} B^{\prime}}+\frac{1}{l} B_{A^{\prime}} \wedge F^{A^{\prime}}\right)+\frac{e^{2}}{2}\left(B_{A^{\prime} B^{\prime}} \wedge B^{A^{\prime} B^{\prime}}+\frac{\mu^{2}}{l} B_{A^{\prime}} \wedge B^{A^{\prime}}\right) \\
& \left.-\lambda^{A^{\prime} B^{\prime}}\left(\frac{1}{l^{2}} e_{A^{\prime} A} \wedge e_{B}^{\prime A}-B_{A^{\prime} B^{\prime}}\right)-\lambda^{A^{\prime}}\left(\frac{1}{l} e_{A^{\prime} A} \wedge \psi^{A}-B_{A^{\prime}}\right)\right\} .
\end{aligned}
$$

We proceed with the $3+1$ decomposition of the action. We assume the spacetime has hyperbolic structure and has the topology of $R \times \Sigma$. We then express the action in terms of space and time independently

$$
\begin{aligned}
& -i \mathcal{I}=-i \int d t \int_{\Sigma} d x^{3} \epsilon^{a b c}\left\{\frac{1}{g^{2}}\left(B_{a b}{ }^{A B} \dot{A}_{c A B}+\frac{1}{l} B_{a b}{ }^{A} \dot{\psi}_{c A}-B_{a b}{ }^{A^{\prime} B^{\prime}} \dot{A}_{c A^{\prime} B^{\prime}}-\frac{1}{l} B_{a b}{ }^{A^{\prime}} \dot{\chi}_{c A^{\prime}}\right)\right. \\
& +\frac{1}{g^{2}} A_{0}^{A B}\left(D_{a} B_{b c A B}+\frac{1}{l} B_{b c A} \psi_{a B}\right)-\frac{1}{g^{2}} A_{0}^{A^{\prime} B^{\prime}}\left(\bar{D}_{a} B_{b c A^{\prime} B^{\prime}}+\frac{1}{l} B_{b c A^{\prime}} \chi_{a B^{\prime}}\right) \\
& +\frac{1}{l} \psi_{0}{ }^{A}\left[\frac{1}{g^{2}}\left(D_{a} B_{b c A}+G \sqrt{\Lambda} B_{b c A B} \psi_{a}^{B}\right)+\lambda^{A^{\prime}}{ }_{a b} e_{c A^{\prime} A}-\frac{1}{g^{2} l} B_{a b}^{A^{\prime}} e_{c A^{\prime} A}\right] \\
& -\frac{1}{l} \chi_{0}{ }^{A^{\prime}}\left[\frac{1}{g^{2}}\left(\bar{D}_{a} B_{b c A^{\prime}}+G \sqrt{\Lambda} B_{b c A^{\prime} B^{\prime}} \chi_{a}^{B^{\prime}}\right)+\lambda^{A}{ }_{a b} e_{c A A^{\prime}}-\frac{1}{g^{2} l} B_{a b}^{A} e_{c A A^{\prime}}\right] \\
& +e_{A A^{\prime} 0}\left[\frac{2}{g^{2} l^{2}} B_{a b}^{A B} e_{c B}^{A^{\prime}}+\frac{2}{l^{2}} \lambda_{a b}^{A B} e_{c B}^{A^{\prime}}+\frac{1}{l} \lambda_{a b}^{A} \chi_{c}^{A^{\prime}}-\frac{1}{g^{2} l^{2}} B_{a b}^{A} \chi_{c}^{A^{\prime}}\right. \\
& \left.-\frac{2}{g^{2} l^{2}} B_{a b}^{A^{\prime} B^{\prime}} e_{c B^{\prime}}^{A}-\frac{2}{l^{2}} \lambda_{a b}^{A^{\prime} B^{\prime}} e_{c B^{\prime}}{ }^{A}-\frac{1}{l} \lambda_{a b}^{A^{\prime}} \psi_{c}^{A}+\frac{1}{g^{2} l^{2}} B_{a b}^{A^{\prime}} \psi_{c}^{A}\right] \\
& +B_{0 a}^{A B}\left[\frac{1}{g^{2}} f_{b c A B}+\frac{1}{g^{2} l^{2}} e_{b A}^{A^{\prime}} e_{c B A^{\prime}}+\frac{1}{g^{2}} \frac{G \sqrt{\Lambda}}{2 l} \psi_{b A} \psi_{c B}-e^{2} B_{b c A B}-\lambda_{b c A B}\right] \\
& -B_{0 a}^{A^{\prime} B^{\prime}}\left[\frac{1}{g^{2}} f_{b c A^{\prime} B^{\prime}}+\frac{1}{g^{2} l^{2}} e_{b A^{\prime}}{ }^{A} e_{c B^{\prime} A}+\frac{1}{g^{2}} \frac{G \sqrt{\Lambda}}{2 l} \chi_{b A^{\prime}} \chi_{c B^{\prime}}-e^{2} B_{b c A^{\prime} B^{\prime}}-\lambda_{b c A^{\prime} B^{\prime}}\right] \\
& +\frac{1}{l} B_{0 a}^{A}\left[\frac{1}{g^{2}} f_{b c A}-\frac{1}{g^{2} l} e_{b A A^{\prime}} \chi_{c}^{A^{\prime}}-e^{2} \mu^{2} B_{b c A}-l \lambda_{b c A}\right] \\
& -\frac{1}{l} B_{0 a}^{A^{\prime}}\left[\frac{1}{g^{2}} f_{b c A^{\prime}}-\frac{1}{g^{2} l} B_{b A^{\prime} A} \psi_{c}^{A}-e^{2} \mu^{2} B_{b c A^{\prime}}-l \lambda_{b c A^{\prime}}\right] \\
& +\lambda_{0 a}^{A B}\left[\frac{1}{l^{2}} e_{b A}^{A^{\prime}} e_{c B A^{\prime}}-B_{b c A B}\right]-\lambda_{0 a}^{A^{\prime} B^{\prime}}\left[\frac{1}{l^{2}} e_{b A^{\prime}}^{A} e_{c B^{\prime} A}-B_{b c A^{\prime} B^{\prime}}\right] \\
& \left.+\lambda_{0 a}^{A}\left[\frac{1}{l} e_{b A}^{A^{\prime}} \chi_{c A^{\prime}}-B_{b c A}\right]-\lambda_{0 a}^{A^{\prime}}\left[\frac{1}{l} e_{b A^{\prime}}^{A} \psi_{c A}-B_{b c A^{\prime}}\right]\right\} \text {. }
\end{aligned}
$$


Here 0 is the time-like index and $a, b, c(=1,2,3)$ are space-like indices. From (27), we define the non-vanishing momenta for the forms $A_{A B}, A_{A^{\prime} B^{\prime}}, \psi_{A}, \chi_{A^{\prime}}$ asf

$$
\begin{array}{cc}
\pi_{A B}^{a}:=\frac{-i}{g^{2}} \epsilon^{a b c} B_{b c A B} & \pi_{A^{\prime} B^{\prime}}^{a}:=\frac{i}{g^{2}} \epsilon^{a b c} B_{b c A^{\prime} B^{\prime}}, \\
\pi_{A}^{a}:=\frac{-i}{g^{2}} \epsilon^{a b c} B_{b c A} & \pi_{A^{\prime}}^{a}:=\frac{i}{g^{2}} \epsilon^{a b c} B_{b c A^{\prime}} .
\end{array}
$$

The other momenta for the forms $B, \lambda$, and $e_{0 A^{\prime} B^{\prime}}$ vanish. We then rewrite the action as

$$
\begin{aligned}
& -i \mathcal{I}=\int d t \int_{\Sigma} d x^{3}\left\{\left(\pi_{A B}^{a} \dot{A}_{a}^{A B}+\frac{1}{l} \pi_{A}^{a} \dot{\psi}_{a}^{A}+\pi_{A^{\prime} B^{\prime}}^{a} \dot{A}_{a}^{A^{\prime} B^{\prime}}+\frac{1}{l} \pi_{A^{\prime}}^{a} \dot{\chi}_{a}^{A^{\prime}}\right)\right. \\
& +A_{0}^{A B}\left(D_{a} \pi_{A B}^{a}+\frac{1}{l} \pi_{A}^{a} \psi_{a B}\right)+A_{0}^{A^{\prime} B^{\prime}}\left(\bar{D}_{a} \pi_{A^{\prime} B^{\prime}}^{a}+\frac{1}{l} \pi_{A^{\prime}}^{a} \chi_{a B^{\prime}}\right) \\
& +\frac{1}{l} \psi_{0}{ }^{A}\left[\left(D_{a} \pi_{A}^{a}+G \sqrt{\Lambda} \pi_{A B}^{a} \psi_{a}^{B}\right)-i \epsilon^{a b c} \lambda^{A^{\prime}}{ }_{a b} e_{c A^{\prime} A}+\frac{1}{l} \pi^{a A^{\prime}} e_{a A^{\prime} A}\right] \\
& -\frac{1}{l} \chi_{0}{ }^{A^{\prime}}\left[\left(\bar{D}_{a} \pi_{A^{\prime}}^{a}+G \sqrt{\Lambda} \pi_{A^{\prime} B^{\prime}}^{a} \chi_{a}^{B^{\prime}}\right)+i \epsilon^{a b c} \lambda^{A}{ }_{a b} e_{c A A^{\prime}}+\frac{1}{l} \pi^{a A} e_{a A A^{\prime}}\right] \\
& +e_{A A^{\prime} 0}\left[\frac{2}{l^{2}} \pi^{a A B} e_{a B}^{A^{\prime}}-\frac{1}{l^{2}} \pi^{a A} \chi_{a}^{A^{\prime}}-\frac{2 i}{l^{2}} \epsilon^{a b c} \lambda_{a b}^{A B} e_{c B}^{A^{\prime}}-\frac{i}{l} \epsilon^{a b c} \lambda_{a b}^{A} \chi_{c}^{A^{\prime}}\right. \\
& \left.+\frac{2}{l^{2}} \pi^{a A^{\prime} B^{\prime}} e_{a B^{\prime}}^{A}-\frac{1}{l^{2}} \pi^{a A^{\prime}} \psi_{a}^{A}+\frac{2 i}{l^{2}} \epsilon^{a b c} \lambda_{a b}^{A^{\prime} B^{\prime}} e_{c B^{\prime}}^{A}+\frac{i}{l} \epsilon^{a b c} \lambda_{a b}^{A^{\prime}} \psi_{c}^{A}\right] \\
& -i B_{0 a}^{A B}\left[\frac{1}{g^{2}} \epsilon^{a b c} f_{b c A B}+\frac{1}{g^{2} l^{2}} \epsilon^{a b c} e_{b A}^{A^{\prime}} e_{c B A^{\prime}}+\frac{1}{g^{2}} \frac{G \sqrt{\Lambda}}{2 l} \epsilon^{a b c} \psi_{A b} \psi_{B c}-i e^{2} g^{2} \pi_{A B}^{a}-\epsilon^{a b c} \lambda_{b c A B}\right] \\
& +i B_{0 a}^{A^{\prime} B^{\prime}}\left[\frac{1}{g^{2}} \epsilon^{a b c} f_{b c A^{\prime} B^{\prime}}+\frac{1}{g^{2} l^{2}} \epsilon^{a b c} e_{b A^{\prime}}^{A} e_{c B^{\prime} A}+\frac{1}{g^{2}} \frac{G \sqrt{\Lambda}}{2 l} \epsilon^{a b c} \chi_{A^{\prime} b} \chi_{B^{\prime} c}+i e^{2} g^{2} \pi_{A^{\prime} B^{\prime}} a-\epsilon^{a b c} \lambda_{b c A^{\prime} B^{\prime}}\right] \\
& -\frac{i}{l} B_{0 a}^{A}\left[\frac{1}{g^{2}} \epsilon^{a b c} f_{b c A}-\frac{1}{g^{2} l} \epsilon^{a b c} e_{b A A^{\prime}} \chi_{c}^{A^{\prime}}-i e^{2} g^{2} \mu^{2} \pi_{A}^{a}-l \epsilon^{a b c} \lambda_{b c A}\right] \\
& +\frac{i}{l} B_{0 a}^{A^{\prime}}\left[\frac{1}{g^{2}} \epsilon^{a b c} f_{b c A^{\prime}}-\frac{1}{g^{2} l} \epsilon^{a b c} e_{b A^{\prime} A} \psi_{c}^{A}+i e^{2} g^{2} \mu^{2} \pi_{A^{\prime}}^{a}-l \epsilon^{a b c} \lambda_{b c A^{\prime}}\right] \\
& -i \lambda_{0 a}^{A B}\left[\frac{1}{l^{2}} \epsilon^{a b c} e_{b A}^{A^{\prime}} e_{c B A^{\prime}}-i g^{2} \pi_{A B}^{a}\right]+i \lambda_{0 a}^{A^{\prime} B^{\prime}}\left[\frac{1}{l^{2}} \epsilon^{a b c} e_{b A^{\prime}}^{A} e_{c B^{\prime} A}+i g^{2} \pi_{A^{\prime} B^{\prime}}^{a}\right] \\
& \left.-i \lambda_{0 a}^{A}\left[\frac{1}{l} \epsilon^{a b c} e_{b A}^{A^{\prime}} \chi_{c A^{\prime}}-i g^{2} \pi_{A}^{a}\right]+i \lambda_{0 a}^{A^{\prime}}\left[\frac{1}{l} \epsilon^{a b c} e_{b A^{\prime}}^{A} \psi_{c A}+i g^{2} \pi_{A^{\prime}}^{a}\right]\right\} \text {. }
\end{aligned}
$$

\subsection{The primary constraints}

We first consider those constraints associated with Lagrange multipliers $\lambda_{0}$. The solutions to these equations will help to simplify the other constraints. To break the topological gauge symmetry of the BF theory, and in the process introduce the local degrees of freedom, additional constraints have been introduced in (27). Their canonical form can be read off of (30), yielding

$$
J_{A B}^{a}:=\frac{1}{l^{2}} \epsilon^{a b c} e_{b A}^{A^{\prime}} e_{c B A^{\prime}}-i g^{2} \pi_{A B}^{a}=0,
$$

\footnotetext{
${ }^{2}$ These will receive corrections when we introduce the boundary terms.
} 


$$
\begin{aligned}
J_{A^{\prime} B^{\prime}}^{a} & :=\frac{1}{l^{2}} \epsilon^{a b c} e_{b A^{\prime}}^{A} e_{c B^{\prime} A}+i g^{2} \pi_{A^{\prime} B^{\prime}}^{a}=0, \\
J_{A}^{a} & :=\frac{1}{l} \epsilon^{a b c} e_{b A}^{A^{\prime}} \chi_{c A^{\prime}}-i g^{2} \pi_{A}^{a}=0, \\
J_{A^{\prime}}^{a} & :=\frac{1}{l} \epsilon^{a b c} e_{b A^{\prime}}^{A} \psi_{c A}+i g^{2} \pi_{A^{\prime}}^{a}=0 .
\end{aligned}
$$

These equations set the $\pi^{a A^{\prime} B^{\prime}}, \pi^{a A B}, \pi^{a A}$ and $\pi^{a A^{\prime}}$ to be the duals of the two forms constructed by the frame fields and the spinor fields.

We first discuss the solution to the bosonic constraints (31) and (32). These are the same as in general relativity and we refer the reader to [2] for more discussion of the following points. We introduce the quantities $N_{A A^{\prime}}$ which is defined as

$$
N_{A A^{\prime}}=t^{\mu} e_{\mu A A^{\prime}}
$$

where $t^{\mu}$ is the timelike unit normal satisfying $t^{\mu} t_{\mu}=-1$. Then we can express $e_{a}^{A A^{\prime}}$, in terms of $N_{B}^{A^{\prime}}$ and either $\pi^{a A B}$ or $\pi^{a A^{\prime} B^{\prime}}$

$$
\begin{aligned}
e_{a}^{A A^{\prime}} & =\frac{1}{\sqrt{h}} \epsilon_{a b c} \pi^{b B C} \pi_{C}^{c A} N_{B}^{A^{\prime}} \\
e_{a}^{A^{\prime} A} & =\frac{1}{\sqrt{h}} \epsilon_{a b c} \pi^{b B^{\prime} C^{\prime}} \pi_{C^{\prime}}^{c A^{\prime}} N_{B^{\prime}}^{A}
\end{aligned}
$$

where $h$ is the determinant of the spatial metric $h_{a b}$.

Notice that there is a secondary constraint, which is

$$
R_{a}^{A A^{\prime}}=\frac{1}{\sqrt{h}} \epsilon_{a b c} \pi^{b B C} \pi_{C}^{c A} N_{B}^{A^{\prime}}-\frac{1}{\sqrt{h}} \epsilon_{a b c} \pi^{b B^{\prime} C^{\prime}} \pi_{C^{\prime}}^{c A^{\prime}} N_{B^{\prime}}^{A}
$$

This expresses the reality conditions. We will later use a consequence of this, which expresses the idea that the areas of surfaces defined from the left handed fields are equal to the areas defined from the right handed fields[2].

We now come to the treatment of the fermion variables. There is a difficulty which arises from equations (33) and (34). These tell us that the Poisson antibracket $\left\{\psi_{A}, \chi_{A^{\prime}}\right\}$ is non-zero. Hence the configuration space can't contain both fermionic fields $\psi_{A}$ and $\chi_{A^{\prime}}$ and we cannot construct a quantum theory in terms of simultaneous eigenstates of $\psi_{A}$ and $\chi_{A^{\prime}}$. This is a well known problem in fermionic theories, there are two ways of handling it. 


\subsection{Treatment of the fermionic constraints: method 1:}

We break the left-right symmetry immediately and choose, arbitrarily to diagonalize one of the fermion fields, say $\psi_{A}$, while treating the other, $\chi_{A^{\prime}}$, as a momentum field. This means that we will choose a representation such that

$$
\hat{\psi}^{A}|\Gamma\rangle=\psi^{A}|\Gamma\rangle
$$

in which the action of $\chi_{A^{\prime}}$ will be

$$
\hat{\chi}^{A^{\prime}}|\Gamma\rangle=e^{A A^{\prime}} \frac{\delta}{\delta \psi^{A}}|\Gamma\rangle
$$

Here for convenience we'd like to choose the conjugate pairs $\left(A_{a}^{A B}, \pi_{A B}^{a}\right),\left(A_{a}^{A^{\prime} B^{\prime}}, \pi_{A^{\prime} B^{\prime}}^{a}\right)$, and $\left(\psi_{a}^{A}, \pi_{A}^{a}\right)$ for the phase space, and the Poisson brackets are

$$
\begin{aligned}
\left\{\pi^{a A B}(x), A_{b C D}(y)\right\} & =\delta_{b}^{a} \delta_{(C}^{A} \delta_{D)}^{B} \delta^{3}(x, y), \\
\left\{\pi^{a A^{\prime} B^{\prime}}(x), A_{b C^{\prime} D^{\prime}}(y)\right\} & =\delta_{b}^{a} \delta_{\left(C^{\prime}\right.}^{A^{\prime}} \delta_{\left.D^{\prime}\right)}^{B^{\prime}} \delta^{3}(x, y), \\
\left\{\pi^{a A}(x), \psi_{b B}(y)\right\} & =-\delta_{b}^{a} \delta_{B}^{A} \delta^{3}(x, y) .
\end{aligned}
$$

with the rest being zero. We need to solve for the other variables $e_{a}^{A A^{\prime}}, \pi^{a A^{\prime}}, \chi_{a}^{A^{\prime}}$ in terms of the canonical momenta from equations (36)-(43). To do this we solve the remaining primary constraints (33) and (34) to find,

$$
\begin{aligned}
\chi_{c}^{A^{\prime}} & =\frac{1}{\sqrt{h}} \frac{1}{l} \epsilon_{a b c} \pi^{a A B} \pi_{A}^{b} N_{B}^{A^{\prime}} \\
\pi_{A^{\prime}}^{a} & =\frac{i}{g^{2} l} \epsilon^{a b c} e_{b A^{\prime}}^{A} \psi_{c A} .
\end{aligned}
$$

Proceeding from here we quickly reach the form of canonical supergravity discussed already by Jacobson in [11].

\subsection{Treatment of the fermionic constraints: method 2:}

The second method is to keep left-right symmetry at the cost of keeping in the theory both sets of fermionic variables, along with the constraints that imply their redundancy. This will be convenient for the study of the boundary theory as well as the quantization. 
To do this we find the secondary constraints which are analogous to (38) which impose the relations between the left and right fermionic variables. Taking linear combinations of (33) and (34) we find that

$$
\begin{aligned}
\psi_{a}^{A} J_{A}^{a} & :=\frac{1}{l} \epsilon^{a b c} \psi_{a}^{A} e_{b A}^{A^{\prime}} \chi_{c A^{\prime}}-i g^{2} \psi_{a}^{A} \pi_{A}^{a}=0 \\
\chi_{a}^{A^{\prime}} J_{A^{\prime}}^{a} & :=\frac{1}{l} \epsilon^{a b c} \chi_{a}^{A^{\prime}} e_{b A^{\prime}}^{A} \psi_{c A}+i g^{2} \chi_{a}^{A^{\prime}} \pi_{A^{\prime}}^{a}=0 .
\end{aligned}
$$

By adding and subtracting we get an equivalent set of constraints

$$
\begin{aligned}
& R=\psi_{a}^{A} \pi_{A}^{a}-\chi_{a}^{A^{\prime}} \pi_{A^{\prime}}^{a}=0 \\
& S^{0}=\epsilon^{a b c} \chi_{a}^{A^{\prime}} e_{b A^{\prime}}^{A} \psi_{c A}=0
\end{aligned}
$$

eliminating $e_{b A^{\prime}}^{A}$ we have

$$
S=\chi_{a}^{A^{\prime}} N_{A^{\prime}}^{B} \pi_{B C}^{[a} \pi^{b] C A} \psi_{b A}=0
$$

\subsection{Elimination of the lagrange multipliers $\lambda$.}

We will want to eliminate the $\lambda_{A B}$ and $\lambda_{A^{\prime} B^{\prime}}$ from the canonical theory. This can be done by solving the constraints that follow from the vanishing of the canonical momenta for $B_{A B}, B_{A^{\prime} B^{\prime}}, B_{A}, B_{A^{\prime}}$. These are

$$
\begin{gathered}
I_{A B}^{a}:=\frac{1}{g^{2}} \epsilon^{a b c} f_{b c A B}+\frac{1}{g^{2} l^{2}} \epsilon^{a b c} e_{b A}^{A^{\prime}} e_{c B A^{\prime}}+\frac{1}{g^{2}} \frac{G \sqrt{\Lambda}}{2 l} \epsilon^{a b c} \psi_{A b} \psi_{B c}-i e^{2} g^{2} \pi_{A B}^{a}-\epsilon^{a b c} \lambda_{b c A B}=0, \\
I_{A^{\prime} B^{\prime}}^{a}:=\frac{1}{g^{2}} \epsilon^{a b c} f_{b c A^{\prime} B^{\prime}}+\frac{1}{g^{2} l^{2}} \epsilon^{a b c} e_{b A^{\prime}}^{A} e_{c B^{\prime} A}+\frac{1}{g^{2}} \frac{G \sqrt{\Lambda}}{2 l} \epsilon^{a b c} \chi_{A^{\prime} b} \chi_{B^{\prime} c}+i e^{2} g^{2} \pi_{A^{\prime} B^{\prime}}^{a}-\epsilon^{a b c} \lambda_{b c A^{\prime} B^{\prime}}=0, \\
I_{A}^{a}:=\frac{1}{g^{2}} \epsilon^{a b c} f_{b c A}-\frac{1}{g^{2} l} \epsilon^{a b c} e_{b A A^{\prime}} \chi_{c}^{A^{\prime}}-i e^{2} g^{2} \mu^{2} \pi_{A}^{a}-l \epsilon^{a b c} \lambda_{b c A}=0 \\
I_{A^{\prime}}^{a}:=\frac{1}{g^{2}} \epsilon^{a b c} f_{b c A^{\prime}}-\frac{1}{g^{2} l} \epsilon^{a b c} e_{b A^{\prime} A} \psi_{c}^{A}+i e^{2} g^{2} \mu^{2} \pi_{A^{\prime}}^{a}-l \epsilon^{a b c} \lambda_{b c A^{\prime}}=0
\end{gathered}
$$

These are second class constraints and can be solved to eliminate $\lambda_{b c A B}, \lambda_{b c A^{\prime} B^{\prime}}, \lambda_{b c A}$, and $\lambda_{b c A^{\prime}}$ in terms of the other variables:

$$
\begin{gathered}
\epsilon^{a b c} \lambda_{b c A B}=\frac{1}{g^{2}} \epsilon^{a b c} f_{b c A B}+i \pi_{A B}^{a}+\frac{1}{g^{2}} \frac{G \sqrt{\Lambda}}{2 l} \epsilon^{a b c} \psi_{b A} \psi_{c B}-i e^{2} g^{2} \pi_{A B}^{a}, \\
\epsilon^{a b c} \lambda_{b c A^{\prime} B^{\prime}}=\frac{1}{g^{2}} \epsilon^{a b c} f_{b c A^{\prime} B^{\prime}}-i \pi_{A^{\prime} B^{\prime}}^{a}+\frac{1}{g^{2}} \frac{G \sqrt{\Lambda}}{2 l} \epsilon^{a b c} \chi_{A^{\prime} b} \chi_{B^{\prime} c}+i e^{2} g^{2} \pi_{A^{\prime} B^{\prime}}^{a}, \\
l \epsilon^{a b c} \lambda_{b c A}=\frac{1}{g^{2}} \epsilon^{a b c} f_{b c A}-i \pi_{A}^{a}-i e^{2} g^{2} \mu^{2} \pi_{A}^{a} \\
l \epsilon^{a b c} \lambda_{b c A^{\prime}}=\frac{1}{g^{2}} \epsilon^{a b c} f_{b c A^{\prime}}+i \pi_{A^{\prime}}^{a}+i e^{2} g^{2} \mu^{2} \pi_{A^{\prime}}^{a} .
\end{gathered}
$$




\subsection{The Gauss and supersymmetry constraints}

Now we come to the constraints that impose the $O s p(1 \mid 2)_{L} \otimes O s p(1 \mid 2)_{R}$ local gauge symmetry. These are

$$
\begin{aligned}
G_{A B} & :=D_{a} \pi_{A B}^{a}+\frac{1}{l} \pi_{A}^{a} \psi_{a B}=0 \\
G_{A}^{L} & :=D_{a} \pi_{A}^{a}+G \sqrt{\Lambda} \pi_{A B}^{a} \psi_{a}^{B}+\frac{1}{l} \pi^{a A^{\prime}} e_{a A^{\prime} A}-i \epsilon^{a b c} \lambda^{A^{\prime}}{ }_{a b} e_{c A^{\prime} A} \\
& =D_{a} \pi_{A}^{a}+G \sqrt{\Lambda} \pi_{A B}^{a} \psi_{a}^{B}+\left(\frac{-i}{g^{2} l} \epsilon^{a b c} f_{b c}^{A^{\prime}}+\frac{G \sqrt{\Lambda}}{3 l} \pi^{a A^{\prime}}\right) e_{a A^{\prime} A}=0 \\
G_{A^{\prime} B^{\prime}} & :=\bar{D}_{a} \pi_{A^{\prime} B^{\prime}}^{a}+\frac{1}{l} \pi_{A^{\prime}}^{a} \chi_{a B^{\prime}}=0 \\
G_{A^{\prime}}^{R} & :=\bar{D}_{a} \pi_{A^{\prime}}^{a}+G \sqrt{\Lambda} \pi_{A^{\prime} B^{\prime}}^{a} \chi_{a}^{B^{\prime}}+\frac{1}{l} \pi^{a A} e_{a A A^{\prime}}+i \epsilon^{a b c} \lambda_{a b}^{A} e_{c A A^{\prime}} \\
& =\bar{D}_{a} \pi_{A^{\prime}}^{a}+G \sqrt{\Lambda} \pi_{A^{\prime} B^{\prime}}^{a} \chi_{a}^{B^{\prime}}+\left(\frac{i}{g^{2 l}} \epsilon^{a b c} f_{b c}^{A}+\frac{G \sqrt{\Lambda}}{3 l} \pi^{a A}\right) e_{a A A^{\prime}}=0 .
\end{aligned}
$$

where (55) (56) are used to cancel the multipliers $\lambda_{a b}^{A}$ and $\lambda_{a b}^{A^{\prime}}$ out of the equations (58) and (60). Here $G_{A B}$ and $G_{A^{\prime} B^{\prime}}$ are nothing but the ordinary $S U(2)_{L} \oplus S U(2)_{R}$ Gauss law constraints, and $G_{A}^{L}, G_{A^{\prime}}^{R}$ are the left-handed and right-handed supersymmetry constraints respectively, which are different from those of chiral supergravity due to the appearance of $e_{A A^{\prime}}$, which mix the left-handed supersymmetry constraints $\left(L_{A}\right)$ and right-handed ones $\left(R_{A^{\prime}}\right)$ in chiral supergravities together in the form:

$$
G_{A}^{L}=L_{A}+\bar{R}_{A} \quad G_{A^{\prime}}^{R}=\bar{L}_{A^{\prime}}+R_{A^{\prime}}
$$

where

$$
\begin{aligned}
L_{A}: & =D_{a} \pi_{A}^{a}-G \sqrt{\Lambda} \pi_{A B}^{a} \psi_{a}^{B} \\
R_{A^{\prime}} & =\left(\frac{-i}{g^{2} l} \epsilon^{a b c} f_{b c}^{A}+\frac{G \sqrt{\Lambda}}{3 l} \pi^{a A}\right) e_{a A A^{\prime}}
\end{aligned}
$$

and $R_{A^{\prime}}$ seems not to be conjugate of $L_{A}$. However, using (36)-(43) and (18), we can change the form of $\bar{R}_{A^{\prime}}$ and find,

$$
\begin{aligned}
\bar{R}_{A} & =\left(\frac{-i}{g^{2} l} \epsilon^{a b c} f_{b c}^{A^{\prime}}+\frac{G \sqrt{\Lambda}}{3 l} \pi^{a A^{\prime}}\right) e_{a A^{\prime} A}=D_{a} \pi_{A}^{a}+\frac{G \sqrt{\Lambda}}{3} \pi_{A B}^{a} \psi_{a}^{B} \\
& =L_{A}-\frac{2 G \sqrt{\Lambda}}{3} \pi_{A B}^{a} \psi_{a}^{B}, \\
\bar{L}_{A^{\prime}} & =\bar{D}_{a} \pi_{A^{\prime}}^{a}+G \sqrt{\Lambda} \pi_{A^{\prime} B^{\prime}}^{a} \psi_{a}^{B^{\prime}}=\left(\frac{i}{g^{2} l} \epsilon^{a b c} f_{b c}^{A}+G \sqrt{\Lambda} \pi^{a A}\right) e_{a A A^{\prime}} \\
& =R_{A^{\prime}}+\frac{2 G \sqrt{\Lambda}}{3 l} \pi^{a A} e_{a A A^{\prime}} .
\end{aligned}
$$

\footnotetext{
${ }^{3}$ This is the characteristic of chiral supergravity, see 11].
} 
To show this, we only need consider the key term and find it can be changed into the following expression,

$$
\begin{aligned}
\frac{-i}{g^{2} l} \epsilon^{a b c} f_{b c}^{A^{\prime}} e_{a A^{\prime} A} & =\frac{-i}{g^{2} l} \epsilon^{a b c}\left[d_{b} \chi_{c}^{A^{\prime}}+A_{b}^{A^{\prime} B^{\prime}} \chi_{c B^{\prime}}\right] e_{a A^{\prime} A} \\
& =\frac{-i}{g^{2} l} \epsilon^{a b c}\left[d_{b} \chi_{c}^{A^{\prime}} e_{a A^{\prime} A}\right]+\frac{-i}{g^{2} l} \epsilon^{a b c} \chi_{c}^{A^{\prime}}\left(d_{b} e_{a A^{\prime} A}+A_{b A^{\prime}}^{B^{\prime}} e_{a B^{\prime} A}\right) \\
& =D_{b} \pi_{A}^{b}-A_{b A}^{B} \pi_{B}^{b}+\frac{-i}{g^{2} l} \epsilon^{a b c} \chi_{c}^{A^{\prime}} \bar{D}_{b} e_{a A^{\prime} A} \\
& =D_{b} \pi_{A}^{b}-A_{b A}^{B} \pi_{B}^{b}+\frac{-i}{g^{2} l} \epsilon^{a b c} \chi_{c}^{A^{\prime}}\left[-A_{b A}^{B} e_{a A^{\prime} B}-\frac{G \sqrt{\Lambda}}{2 l} \chi_{b A^{\prime}} \psi_{a A}\right] \\
& =D_{b} \pi_{A}^{b}-\frac{i \sqrt{\Lambda}}{2} \epsilon^{a b c} \chi_{c}^{A^{\prime}} \chi_{b A^{\prime}} \psi_{a A} \\
& =D_{b} \pi_{A}^{b} .
\end{aligned}
$$

Combining these equations together we find:

$$
\begin{aligned}
G_{A}^{L} & =2\left(D_{a} \pi_{A}^{a}+\frac{2 G \sqrt{\Lambda}}{3} \pi_{A B}^{a} \psi_{a}^{B}\right)=0, \\
G_{A^{\prime}}^{R} & =2\left(\frac{i}{g^{2} l} \epsilon^{a b c} f_{b c}^{A}+\frac{2 G \sqrt{\Lambda}}{3 l} \pi^{a A}\right) e_{a A A^{\prime}}=0 .
\end{aligned}
$$

They are conjugate of each other indeed! We may also note that if we write

$$
G_{A^{\prime}}^{R}=\frac{N_{A^{\prime}}^{A}}{\sqrt{h}} G_{A}^{R}
$$

we have

$$
G_{A}^{R}=2\left(\frac{i}{g^{2} l} f_{b c}^{B}+\frac{2 G \sqrt{\Lambda}}{3 l} \pi^{a B} \epsilon_{a b c}\right) \pi_{B D}^{b} \pi^{c D A} .
$$

If we consider the Poisson brackets between the $G_{A B}$ and $G_{A}^{L}$, we find that they does form a close algebra for $O s p(1 \mid 2)$ as in the case of chiral supergravity

$$
\begin{gathered}
\left\{G(\lambda), G\left(\lambda^{\prime}\right)\right\}=G\left(\left[\lambda, \lambda^{\prime}\right]\right), \\
\left\{G^{L}(\eta), G(\lambda)\right\}=G^{L}([\eta, \lambda]), \\
\left\{G^{L}(\eta), G^{L}\left(\eta^{\prime}\right)\right\}=G\left(\left[\eta, \eta^{\prime}\right]\right),
\end{gathered}
$$

where $G$ and $G^{L}$ are the constrained functional smeared on the three dimensional space. The same is true for the combination of the $G_{A^{\prime} B^{\prime}}$ and $G_{A^{\prime}}$. So all these equations form the super Gauss's law which generates the $O s p(1 \mid 2)_{L} \oplus O s p(1 \mid 2)_{R}$ gauge transformations of the canonical variables. 
In this sense we can say our model is combining two copies of the chiral supergravities into one.

We still have to discuss the commutator of $G^{A}$ with $G^{A^{\prime}}$. This requires more work as it involves the hamiltonian and diffeomorphism constraints.

\subsection{The hamiltonian and diffeomorphism Constraints}

We come finally to the constraint associated with the Lagrangian multiplier $e_{0 A A^{\prime}}$. As in [2] these will contain the Hamiltonian and diffeomorphism constraints. We have

$$
\begin{aligned}
G^{A A^{\prime}}:= & \frac{2}{l^{2}} \pi^{a A B} e_{a B}^{A^{\prime}}-\frac{1}{l^{2}} \pi^{a A} \chi_{a}^{A^{\prime}}-\frac{2 i}{l^{2}} \epsilon^{a b c} \lambda_{a b}^{A B} e_{c B}^{A^{\prime}}-\frac{i}{l} \epsilon^{a b c} \lambda_{a b}^{A} \chi_{c}^{A^{\prime}} \\
& +\frac{2}{l^{2}} \pi^{a A^{\prime} B^{\prime}} e_{a B^{\prime}}^{A}-\frac{1}{l^{2}} \pi^{a A^{\prime}} \psi_{a}^{A}+\frac{2 i}{l^{2}} \epsilon^{a b c} \lambda_{a b}^{A^{\prime} B^{\prime}} e_{c B^{\prime}}^{A}+\frac{i}{l} \epsilon^{a b c} \lambda_{a b}^{A^{\prime}} \psi_{c}^{A} \\
= & 0 .
\end{aligned}
$$

To understand this constraint better, we try to change it into a new form in which $G_{A A^{\prime}}$ is divided into two conjugate parts $\left(C_{A B}, C_{A^{\prime} B^{\prime}}\right)$.

$$
G^{A A^{\prime}}=\frac{2}{G}\left(C^{A B} \frac{N_{B}^{A^{\prime}}}{\sqrt{h}}+C^{A^{\prime} B^{\prime}} \frac{N_{B^{\prime}}^{A}}{\sqrt{h}}\right) .
$$

To find the forms of $C^{A B}$ and $C^{A^{\prime} B^{\prime}}$ we need only to plug all the solutions (36)-(43) and (53)-(56) into (73), then we find $C^{A B}$ and $C^{A^{\prime} B^{\prime}}$ have the following form

$$
\begin{gathered}
C^{A B}=\frac{G^{2} \Lambda}{3} \epsilon_{a b c} \pi^{a A C} \pi^{b B D} \pi_{C D}^{c}-2 i \pi^{a B C} \pi_{D C}^{b} f_{a b}^{A D}-i \frac{G \sqrt{\Lambda}}{l} \pi^{a B C} \pi_{D C}^{b} \psi_{a}^{A} \psi_{b}^{D} \\
+\frac{G \sqrt{\Lambda}}{3} g^{2} \epsilon_{a b c} \pi^{a A} \pi^{b B C} \pi_{C}^{c}-2 i \pi^{a B C} \pi_{C}^{b} f_{a b}^{A}, \\
C^{A^{\prime} B^{\prime}=} \frac{G^{2} \Lambda}{3} \epsilon_{a b c} \pi^{a A^{\prime} C^{\prime}} \pi^{b B^{\prime} D^{\prime}} \pi_{C^{\prime} D^{\prime}}^{c}+2 i \pi^{a B^{\prime} C^{\prime}} \pi_{D^{\prime} C^{\prime}}^{b} f_{a b}^{A^{\prime} D^{\prime}}+i \frac{G \sqrt{\Lambda}}{l} \pi^{a B^{\prime} C^{\prime}} \pi_{D^{\prime} C^{\prime}}^{b} \chi_{a}^{A^{\prime}} \chi_{b}^{D^{\prime}} \\
+\frac{G \sqrt{\Lambda}}{3} g^{2} \epsilon_{a b c} \pi^{a A^{\prime}} \pi^{b B^{\prime} C^{\prime}} \pi_{C^{\prime}}^{c}+2 i \pi^{a B^{\prime} C^{\prime}} \pi_{C^{\prime}}^{b} f_{a b}^{A^{\prime}} .
\end{gathered}
$$

As is the case in general relativity [2], it turns out that the four constraints $C^{A B}$ contain both the diffeomorphism and hamiltonian constraints of the supergravity. If we only consider the terms only involving the variables $\pi_{A B}^{a}$ and $F_{a b A B}$, then the constraints go back to those of the quantum general relativity [2]. 


\section{The boundary theory}

We are now ready to focus on the construction of the boundary theory. We follow the formulation given in [2] for general relativity with Lorentzian signature. This is related to the Euclidean formulation given in [1], but differs from that in several significant aspects.

We study first the addition of a boundary to the action and equations of motion. Once this is understood we will proceed to their expression in the Hamiltonian formulation.

\subsection{Boundary terms in the action and equations of motion}

In the presence of the boundary, the ordinary variations of the action are not well defined unless boundary terms are added to the action and some boundary conditions are imposed. These must be chosen so as to cancel the boundary term in the variation of the action, so that the total action is functionally differentiable. We will assume that $\mathcal{M}$ has the topology of $R \times \Sigma$, so that $\partial \mathcal{M} \approx R \times \partial \Sigma$, where $\partial \Sigma$ is a compact two manifold. We will assume here that $\partial \Sigma \approx S^{2}$, but it is not difficult to consider the more general case.

It's very interesting that the total action (20) can be supplemented by the $O s p(1 \mid 2)_{L} \oplus$ $O s p(1 \mid 2)_{R}$ super Chern-Simons term with the appropriate boundary condition

$$
\begin{aligned}
S_{c s}= & \frac{i k}{4 \pi} \int_{\partial M} Y_{c s}\left(A_{A B}, \psi_{A}\right)-\frac{i k}{4 \pi} \int_{\partial M} Y_{c s}\left(A_{A^{\prime} B^{\prime}}, \chi_{A^{\prime}}\right) \\
= & \frac{i k}{4 \pi} \int_{\partial M}\left(A^{A B} \wedge \tilde{F}_{A B}+\frac{2}{3} A \wedge A \wedge A+\frac{G \sqrt{\Lambda}}{2 l} \psi^{A} \wedge \tilde{F}_{A}\right) \\
& -\frac{i k}{4 \pi} \int_{\partial M}\left(A^{A^{\prime} B^{\prime}} \wedge \tilde{F}_{A^{\prime} B^{\prime}}+\frac{2}{3} A^{\prime} \wedge A^{\prime} \wedge A^{\prime}+\frac{G \sqrt{\Lambda}}{2 l} \chi^{A^{\prime}} \wedge \tilde{F}_{A^{\prime}}\right),
\end{aligned}
$$

Where $\left(\tilde{F}_{A B}, \tilde{F}_{A}\right)$ and $\left(\tilde{F}_{A^{\prime} B^{\prime}}, \tilde{F}_{A^{\prime}}\right)$ are components of the curvature of the one form connection of $O s p(1 \mid 2)_{L}$ and $O s p(1 \mid 2)_{R}$ respectively. They are of the form

$$
\begin{aligned}
\tilde{F}_{A B} & =f_{A B}+\frac{G \sqrt{\Lambda}}{2 l} \psi_{A} \wedge \psi_{B}, \\
\tilde{F}_{A} & =D \psi_{A}=f_{A} \\
\tilde{F}_{A^{\prime} B^{\prime}} & =f_{A^{\prime} B^{\prime}}+\frac{G \sqrt{\Lambda}}{2 l} \chi_{A^{\prime}} \wedge \chi_{B^{\prime}}, \\
\tilde{F}_{A^{\prime}} & =\bar{D} \chi_{A^{\prime}}=f_{A^{\prime}} .
\end{aligned}
$$

If we take the variation of the total action

$\delta S_{\text {tot }}=\delta S_{B F}+\delta S_{C S}$ 


$$
\begin{aligned}
& =\int_{M}(\ldots)+\int_{\partial M} \delta A^{A B} \wedge\left(\frac{1}{g^{2}} B_{A B}-\frac{k}{2 \pi} \tilde{F}_{A B}\right)+\delta \psi^{A} \wedge\left[\frac{1}{g^{2}} B_{A}-\frac{k}{2 \pi} \frac{G \sqrt{\Lambda}}{2}\left(\tilde{F}_{A}+A_{A B} \wedge \psi^{B}\right)\right] \\
& \left.-\int_{\partial M} \delta A^{A^{\prime} B^{\prime}} \wedge\left(\frac{1}{g^{2}} B_{A^{\prime} B^{\prime}}-\frac{k}{2 \pi} \tilde{F}_{A^{\prime} B^{\prime}}\right)+\delta \chi^{A^{\prime}} \wedge\left[\frac{1}{g^{2}} B_{A^{\prime}}-\frac{k}{2 \pi} \frac{G \sqrt{\Lambda}}{2}\left(\tilde{F}_{A^{\prime}}+A_{A^{\prime} B^{\prime}} \wedge \chi^{B^{\prime}}\right)\right\}, 79\right)
\end{aligned}
$$

we require that the boundary terms vanish. In order to induce a local boundary theory, we require that the integrand of the boundary term in the variation vanish. This leads to the following conditions,

$$
\begin{gathered}
\epsilon^{a b c} \delta A_{c}^{A B}\left(\frac{1}{g^{2}} B_{a b A B}-\frac{k}{2 \pi} \tilde{F}_{a b A B}\right)=0 \\
\epsilon^{a b c} \delta \psi_{c}^{A}\left[\frac{1}{g^{2}} B_{a b A}-\frac{k}{2 \pi} \frac{G \sqrt{\Lambda}}{2}\left(\tilde{F}_{a b A}+A_{a A B} \wedge \psi_{b}^{B}\right)\right]=0 \\
\epsilon^{a b c} \delta A_{c}^{A^{\prime} B^{\prime}}\left(\frac{1}{g^{2}} B_{a b A^{\prime} B^{\prime}}-\frac{k}{2 \pi} \tilde{F}_{a b A^{\prime} B^{\prime}}\right)=0 \\
\epsilon^{a b c} \delta \chi_{a}^{A^{\prime}}\left[\frac{1}{g^{2}} B_{a b A^{\prime}}-\frac{k}{2 \pi} \frac{G \sqrt{\Lambda}}{2}\left(\tilde{F}_{a b A^{\prime}}+A_{a A^{\prime} B^{\prime}} \wedge \chi_{b}^{B^{\prime}}\right)\right]=0 .
\end{gathered}
$$

There are several ways to satisfy these conditions. Taking the boundary term involving $\delta A_{A B}$ as an example, we find

- One can fix the variable $\mathcal{A}_{a}$ on the boundary:

$$
\left.\delta A_{a}^{A B}\right|_{\partial \mathcal{M}}=0
$$

- One can take the self-dual conditions for the curvature $\tilde{F}_{a b A B}$ :

$$
\left(\tilde{F}_{a b A B}-\frac{2 \pi}{g^{2} k} B_{a b A B}\right)_{\partial \mathcal{M}}=0 .
$$

- We can consider some combination of these two conditions in which (84) is imposed on some components of the connection while (85) is imposed on the others.

The first case is simple, but leads to a reduction of the local gauge invariance on the boundary. The next simplest case would be the second (85). This is what was done for the Euclidean theory in [1]. An advantage of this condition is that it leads to no reduction of the local gauge symmetry on the boundary. However, in the Lorentzian case, (85) is too strong, as it can be shown that all of its solutions correspond to a surface wiggling in a fixed $\operatorname{AdS}$ 
space time. By counting there is one degree of freedom, but this turns out to correspond to local longitudinal motions of the surface.

This problem does not arise in the Euclidean case studied in 11$]$ because the left and right handed components of the connection are independent. It is a consequence of the fact that in the Lorentzian theory they are instead related by the reality conditions, which require that they are complex conjugates of each other.

If our boundary conditions are to allow an infinite dimensional space of solutions we must then loosen the self-dual boundary conditions. We do this as follows.

We begin by imposing a time slicing of the boundary, which corresponds to some choice of time coordinate $t$ on $\partial \mathcal{M}$. We then restrict attention to a spatial slice of the boundary at fixed $t=$ constant. We proceed by specifying a set of preferred points on the spatial boundary, which will be called punctures. They will correspond to points where the spin network states meet the boundary in the quantum theory. Each puncture is surrounded by a region of the spatial boundary. In each region we introduce local coordinates $(r, \theta)$ which are angular coordinates with the puncture at the origin. These can then be joined yielding a single coordinate patch on the whole punctured sphere, which reduces to an angular coordinate system in the neighborhood of each puncture. Bringing back $t$ we then have a coordinate system $(r, \theta, t)$ on the whole of $\partial M$ minus the world lines of the punctures.

Then we put the following conditions for the components of the fields

$$
\begin{gathered}
\frac{1}{g^{2}} B_{r \theta A B}-\frac{k}{2 \pi} \tilde{F}_{r \theta A B}=0 \\
\frac{1}{g^{2}} B_{\theta t A B}-\frac{k}{2 \pi} \tilde{F}_{\theta t A B}=0 \\
\delta A_{\theta}=0
\end{gathered}
$$

The first two equations, (86) and (87) correspond to imposing the self-dual boundary conditions on the components $F_{r \theta}$ and $F_{t \theta}$. But instead of constraining the $F_{r t}$ component we impose the third condition (88). The radial component of the connection, $\mathcal{A}_{r}$, is thus unrestricted and is free to evolve with time.

It can be checked that when these conditions have been imposed the bulk theory is not trivial and has an infinite number of solutions. 
We can then write the boundary conditions in terms of the component fields, yielding,

$$
\begin{gathered}
f_{r \theta A B}=\frac{1}{l^{2}} \frac{2 \pi}{g^{2} k} e_{r A A^{\prime}} \wedge e_{\theta B}^{A^{\prime}}-\frac{G \sqrt{\Lambda}}{2 l} \psi_{r A} \wedge \psi_{\theta B}, \\
f_{r \theta A}=\frac{1}{l} \frac{2 \pi}{g^{2} k} e_{r A A^{\prime}} \wedge \chi_{\theta}^{A^{\prime}}, \\
f_{t \theta A B}=\frac{1}{l^{2}} \frac{2 \pi}{g^{2} k} e_{t A A^{\prime}} \wedge e_{\theta B}^{A^{\prime}}-\frac{G \sqrt{\Lambda}}{2 l} \psi_{t A} \wedge \psi_{\theta B}, \\
f_{t \theta A}=\frac{1}{l} \frac{2 \pi}{g^{2} k} e_{t A A^{\prime}} \wedge \chi_{\theta}^{A^{\prime}}, \\
\delta A_{\theta A B}=0 \quad \delta \psi_{\theta A}=0 .
\end{gathered}
$$

Consistency with the field equations leads to relationship between the constant $k$ and the cosmological constant,

$$
G^{2} \Lambda=\frac{6 \pi}{k}
$$

\subsection{Boundary terms in the canonical theory}

Next we describe the expression of the boundary terms and conditions on the canonical theory. The first effect to take into account is that the boundary terms alter the definition of the momenta of the fields, due to the fact that (77) contains time derivatives,

$$
\begin{aligned}
\pi_{A B}^{a} & :=\frac{-i}{g^{2}} \epsilon^{a b c} B_{b c A B}(x)+\frac{i k}{4 \pi} \int d^{2} S(\sigma) \epsilon^{a b} A_{b}^{A B} \delta^{3}(x, S(s)), \\
\pi_{A^{\prime} B^{\prime}}^{a} & :=\frac{i}{g^{2}} \epsilon^{a b c} B_{b c A^{\prime} B^{\prime}}(x)+\frac{-i k}{4 \pi} \int d^{2} S(\sigma) \epsilon^{a b} A_{b}^{A^{\prime} B^{\prime}} \delta^{3}(x, S(s)), \\
\pi_{A}^{a} & :=\frac{-i}{g^{2}} \epsilon^{a b c} B_{b c A}(x)+\frac{i k}{4 \pi} \int d^{2} S(\sigma) \epsilon^{a b} \frac{G \sqrt{\Lambda}}{2 l} \psi_{b}^{A} \delta^{3}(x, S(s)), \\
\pi_{A^{\prime}}^{a} & :=\frac{i}{g^{2}} \epsilon^{a b c} B_{b c A^{\prime}}(x)+\frac{-i k}{4 \pi} \int d^{2} S(\sigma) \epsilon^{a b \frac{G \sqrt{\Lambda}}{2 l}} \chi_{b}^{A^{\prime}} \delta^{3}(x, S(s)),
\end{aligned}
$$

where $\epsilon^{a b}$ is the area element induced by the volume element on the surface with

$$
\epsilon^{a b}:=\epsilon^{a b c} n_{c}
$$

The Gaussian constraints can be expressed as:

$$
G^{A B}=D_{a} p^{a A B}+q^{A B}
$$


Where

$$
p^{a A B}:=\frac{-i}{g^{2}}{ }^{a b c} B_{b c}^{A B}(x)+\frac{i k}{2 \pi} \int d^{2} S(\sigma) \epsilon^{a b} A_{b}^{A B} \delta^{3}(x, S(s)),
$$

and

$$
q^{A B}:=\frac{i}{g^{2} l} \epsilon^{a b c} B_{a b}^{A} \psi_{c}^{B}+\frac{i k}{2 \pi} \int d^{2} S(\sigma) \epsilon^{a b} \frac{G \sqrt{\Lambda}}{2 l} \psi_{a}^{A} \psi_{b}^{B} \delta^{3}(x, \delta(s)) .
$$

The Gauss functional can be obtained by smearing the constraint on the spatial manifold with the appropriate boundary terms,

$$
\begin{aligned}
G\left(\Lambda_{A B}\right)= & \int_{\Sigma} \Lambda_{A B} G^{A B}=\int_{\Sigma} \Lambda_{A B}\left[D_{a}\left(\frac{-i}{g^{2}} \epsilon^{a b c} B_{b c}^{A B}\right)-\frac{-i}{g^{2} l} \epsilon^{a b c} B_{a b}^{A} \psi_{c}^{B}\right] \\
& -\frac{i k}{2 \pi} \int_{\Sigma} \Lambda_{A B} \int d^{2} S(\sigma) \epsilon^{a b} D_{a} A_{b}^{A B} \delta^{3}(x, S(s)) \\
& +\frac{i k}{2 \pi} \int_{\Sigma} \Lambda_{A B} \int d^{2} S(\sigma) \epsilon^{a b} \frac{G \sqrt{\Lambda}}{2 l} \psi_{a}^{A} \psi_{b}^{B} \delta^{3}(x, S(s)) \\
= & -\int_{\Sigma} \frac{i}{g^{2}} \epsilon^{a b c} B_{b c}^{A B} D_{a} \Lambda_{A B}-\int_{\Sigma} \Lambda_{A B} \frac{-i}{g^{2} l} \epsilon^{a b c} B_{a b}^{A} \psi_{a}^{B} \\
& +\int_{\partial \Sigma} d s \Lambda_{A B}\left[\frac{-i}{g^{2}} \epsilon^{a b c} B_{b c}^{A B} n_{a}+\frac{i k}{2 \pi} \epsilon^{a b} f_{a b}^{A B}+\frac{i k}{2 \pi} \frac{G \sqrt{\Lambda}}{2 l} \epsilon^{a b} \psi_{a}^{A} \psi_{b}^{B}\right]
\end{aligned}
$$

In the canonical theory the constraint then has a boundary term:

$$
\left.G\left(\Lambda_{A B}\right)\right|_{b}=\int_{\partial \Sigma} d s \Lambda_{A B}\left[\frac{-i}{g^{2}} \epsilon^{a b c} B_{b c}^{A B} n_{c}+\frac{i k}{2 \pi} \epsilon^{a b}\left(f_{a b}^{A B}+\frac{G \sqrt{\Lambda}}{2 l} \psi_{a}^{A} \psi_{b}^{B}\right)\right] .
$$

This leads to the canonical form of the boundary terms.

$$
\frac{1}{g^{2}} B_{a b A B}=\frac{k}{2 \pi} \tilde{F}_{a b A B} .
$$

Notice that this corresponds with one of the components of the boundary terms we imposed in the lagrangian theory (86). The boundary conditions (87) do not arise in the same way, as they involve the time components of fields, which become lagrange multipliers in the canonical theory. The last components of the lagrangian theory (88) persist in the Hamiltonian theory. These in fact correspond to the fact that in the quantum theory the labels of the punctures are fixed and do not evolve in time.

In a similar way, the boundary term on the supersymmetric constraints can be derived as follows:

$$
G^{A}=D_{a} p^{a A}+q^{A}
$$

where

$$
p^{a A}:=\frac{-i}{g^{2}} \epsilon^{a b c} B_{b c}^{A}(x)+\frac{i k}{2 \pi} \int d^{2} S(\sigma) \epsilon^{a b} \frac{G \sqrt{\Lambda}}{2 l} \psi_{b}^{A} \delta^{3}(x, S(s)),
$$




$$
q^{A}:=\frac{i}{g^{2} l} \frac{2 G \sqrt{\Lambda}}{3 l} \epsilon^{a b c} B_{a b}^{A B} \psi_{c B}+\frac{i k}{2 \pi} \int d^{2} S(\sigma) \epsilon^{a b} \frac{G \sqrt{\Lambda}}{2 l} A_{a}^{A B} \psi_{b B} \delta^{3}(x, S(s)),
$$

and the supersymmetric functional is

$$
\begin{aligned}
G\left(\Lambda_{A}\right)= & \int_{\Sigma} \Lambda_{A} G^{A}=\int_{\Sigma} \Lambda_{A}\left[D_{a}\left(\frac{-i}{g^{2}} \epsilon^{a b c} B_{b c}^{A}\right)-\frac{-i}{g^{2} l} \frac{2 G \sqrt{\Lambda}}{3 l} \epsilon^{a b c} B_{a b}^{A B} \psi_{c B}\right] \\
& -\frac{i k}{2 \pi} \frac{G \sqrt{\Lambda}}{2 l} \int_{\Sigma} \Lambda_{A} \int d^{2} S(\sigma) \epsilon^{a b} D_{a} \psi_{b}^{A} \delta^{3}(x, S(s)) \\
& +\frac{i k}{2 \pi} \int_{\Sigma} \Lambda_{A} \int d^{2} S(\sigma) \epsilon^{a b \frac{G \sqrt{\Lambda}}{2 l}} A_{b}^{A B} \psi_{a B} \delta^{3}(x, S(s)) \\
= & -\int_{\Sigma} \frac{-i}{g^{2}} \epsilon^{a b c} B_{b c}^{A} D_{a} \Lambda_{A}-\int_{\Sigma} \Lambda_{A} \frac{-i}{g^{2} l} \frac{2 G \sqrt{\Lambda}}{3 l} \epsilon^{a b c} B_{a b}^{A B} \psi_{c B} \\
& +\int_{\partial \Sigma} d s \Lambda_{A}\left[\frac{-i}{g^{2}} \epsilon^{a b c} B_{b c}^{A} n_{a}-\frac{i k}{2 \pi} \frac{G \sqrt{\Lambda}}{2 l} \epsilon^{a b}\left(f_{a b}^{A}+A_{a}^{A B} \psi_{b B}\right)\right] .
\end{aligned}
$$

Comparing (109) with (81), we have

$$
\frac{1}{g^{2}} B_{a b A}=\frac{k}{2 \pi} \frac{G \sqrt{\Lambda}}{2}\left(\tilde{F}_{a b A}+A_{a A B} \wedge \psi_{b}^{B}\right) .
$$

From above consideration, we find both Gauss constraint and the supersymmetric constraint are well defined on the spatial surface if we impose the self-dual conditions for the curvatures.

\section{Quantization of the theory}

We are finally ready to discuss the quantization of supergravity, in the presence of boundary conditions (86-88). We follow the methods of loop quantum gravity [6, 7, 8, 9, 10, extended to theories with finite boundaries in [1, 2]. Two important parts of the theory are the spin network basis [6, 7] which we extended to supergravity in [3], and the ChernSimons state [19, 21], which was extended to supergravity in 13]. The combination yields framed or quantum-deformed supersymmetric spin networks, which are constructed from the representation theory of quantum deformed $O s p(1 \mid 2)$ [27].

The method of treating the relationship between the boundary and bulk theory we use

was developed in [1] and then extended to theories with Lorentzian signature in [2]. Here we describe only the results, the reader is referred to [1] and [2] for detail.

There is a well known non-perturbative procedure to deal with the quantization of the bulk theory in the case of general relativity [6, 0, 8, 9, 10]. A set of complete but independent basis of the quantum states can be constructed by means of spin networks. It is a trivalent 
graph $\Gamma$ in which each node join some links. Associated every link, there is a spin $j_{i}$ which satisfies some conditions at the node, which is also labeled by an intertwiner $\nu_{e}$. Recently we extended these techniques to the $\mathrm{N}=1$ chiral supergravity with super Lie algebra $O s p(1 \mid 2)$ [3]. It turns out that an analogous supersymmetric spin networks can be established for the theory. In particular in the basis of super spin networks the spectrum of the area operator can be computed out and partly diagonalized. For tri-valent spin networks its eigenvalues have a discrete form given by [3]:

$$
\hat{A}\left|\Gamma^{s g}, n_{i}, v_{e}\right\rangle=\sum_{i} l_{p}^{2} \sqrt{j_{i}\left(j_{i}+\frac{1}{2}\right)}\left|\Gamma^{s g}, n_{i}, v_{e}\right\rangle .
$$

Where $l_{p}$ is the Planck length and $j_{i}=\frac{n_{i}}{2}$. We need modify the construction slightly to fit the constrained super $B F$ theory, which double covers over the chiral supergravity. The configuration space of the theory is the modular space of $O s p(1 \mid 2) \oplus O \operatorname{sp}(1 \mid 2)$. The basis for the Hilbert space can be constructed in terms of the super spin networks for the super algebra $O s p(1 \mid 2)_{L} \oplus O \operatorname{sp}(1 \mid 2)_{R}$. Then the links of the networks are labeled by conjugate pairs of $\operatorname{spins}\left(j_{L}, j_{R}\right)$ and the nodes are labeled by pairs of intertwiners $\left(\nu_{L}, \nu_{R}\right)$. There is a similar case as in quantum general relativity that we need impose the balanced conditions to the pairs of $\left(j_{L}, j_{R}\right)$ and $\left(\nu_{L}, \nu_{R}\right)$ to coincide with the reality condition of the theory. These conditions are $j_{L}=j_{R}$ and $\nu_{L}=\nu_{R}$.

All the construction above can be extended without difficulty to the quantum deformed version of the superalgebra, $U_{q}(O s p(1 \mid 2))$, whose representation are finite dimensional and bounded by the generic $q$. f The q-deformed formalism of the supergroup give some modification to the spectrum of the area but having a similar expression as the ordinary one,

$$
\hat{A}\left|\Gamma^{s g}, n_{i}, v_{e}\right\rangle=\sum_{i} l_{p}^{2} \sqrt{\left[j_{i}\right]\left[j_{i}+\frac{1}{2}\right]}\left|\Gamma^{s g}, n_{i}, v_{e}\right\rangle,
$$

where

$$
[j]:=\frac{q^{j}-q^{-j}}{q-q^{-1}}
$$

If we impose all the constraints on the state space quantum mechanically and require the results of their action vanish, we could find a set of physical states which actually is the

\footnotetext{
${ }^{4}$ The quantum superalgebra and supergroups $U_{q}(\operatorname{osp}(1 \mid 2))$ are investigated in several places 27 . and its structure of finite dimensional representation are well established.
} 
super Chern-Simons states,

$$
\Psi(\Gamma)=\int D \mathcal{A} e^{\frac{i k}{4 \pi}\left(Y_{c s}\left(A_{A B}, \psi_{A}\right)-Y_{c s}\left(A_{A^{\prime} B^{\prime}}, \chi_{A^{\prime}}\right)\right)}
$$

It allows us to testify the Bekenstein bound in a quantum mechanical sense. We consider the spatial n-punctured boundary with finite area. Associated to every puncture, there is a half-integer $j_{\alpha}$ which takes values from $1 / 2$ to $k / 2$, where $\mathrm{k}$ is the coupling constant of the Chern-Simons theory. Then the total Hilbert space is constructed by the direct sum of the state spaces of all $O s p(1 \mid 2) \oplus O s p(1 \mid 2)$ Chern-Simons theories. Here we pay more attention to the contribution of the boundaries. As we know, for every set of the punctures the Hilbert space of the topological field theory has finite number of degrees of freedom. In our case it is contributed by the conformal blocks for the n punctures of the $O s p(1 \mid 2) \oplus O s p(1 \mid 2) \mathrm{WZW}$ model. We find:

$$
\mathcal{H}_{j_{1}, \ldots, j_{n}}=\mathcal{V}_{j_{1}, \ldots, j_{n}}^{L} \bigoplus \mathcal{V}_{j_{1}, \ldots, j_{n}}^{R}
$$

and the dimensions of the space is:

$$
\operatorname{dim}\left(\mathcal{V}_{j_{1}, \ldots, j_{n}}^{L} \bigoplus \mathcal{V}_{j_{1}, \ldots, j_{n}}^{R}\right)=\prod_{i}\left(4 j_{i}+1\right)
$$

Then based on this result and the holography hypothesis, we can construct the total physical state space as the summation of all the possible states of the topological field theories:

$$
\mathcal{H}^{\text {phys }}=\sum_{n} \sum_{j_{1}, \ldots, j_{n}} \mathcal{H}_{j_{1}, \ldots, j_{n}}
$$

Using (111) and (116), and choosing the constant c, we find the Bekenstein bound is satisfied:

$$
\operatorname{dim}\left(\mathcal{V}_{j_{1}, \ldots, j_{n}}^{L} \bigoplus \mathcal{V}_{j_{1}, \ldots, j_{n}}^{R}\right) \leq \exp \left\{\frac{c A\left[j_{1}, \ldots, j_{n}\right]}{G \hbar}\right\}
$$

\section{Conclusions}

In this paper we have extended to the case of $N=1$ supergravity the basic facts about finite area boundary conditions worked out previously for quantum general relativity in [1, 2]. Here we have treated the case of timelike boundaries following [2], it is straightforward also to find the same results for the Euclidean theory with cosmological constant by restricting to 
the left handed sector and then Euclideanizing, as in [1]. A natural extension of the ChernSimons boundary conditions studied in [1] to null boundaries, suitable for the descriptions of horizons, has been worked out in [29], following observations made in [30]. Following the results given here it should be straightforward also to extend these results to supergravity. Another important thing yet to be done is the construction of quasi-local operators on the boundary which represent the hamiltonian and supersymmetry charges. It may be hoped that this may resolve issues concerning the positivity of the Hamiltonian in loop quantum gravity 31].

The next works in this series will extend these results to $N=2$ supergravity, which should make possible the detailed description of BPS states and BPS black holes in the langauge of loop quantum gravity. This should make possible the direct comparison of the results on black hole state counting coming from string theory and loop quantum gravity. Another application of these results may be to an explicit construction of the boundary conformal field theory in the AdS/CFT conjecture in $3+1$ dimensions.

As a final remark we emphasize the natural way in which the Bekenstein bound is satisfied in quantum general relativity given only the imposition of the appropriate ChernSimons boundary conditions [1]. We see here that this extends naturally to supergravity. This appears to make possible formulations of the holographic principle at the background independent level 32, 33, 34, 35].

\section{ACKNOWLEDGEMENTS}

We are grateful to Abhay Asthtekar, Roger Penrose, Michael Reisenberger and Carlo Rovelli for comments on various aspects of this work. Also both of us would like to thank the theoretical physics group at Imperial college for their hospitality during this last year. This work is supported by the NSF through grant PHY95-14240 and a gift from the Jesse Phillips Foundation.

\section{References}


[1] L. Smolin, Linking topological quantum field theory and nonperturbative quantum gravity, J. Math. Phys. 36(1995)6417, gr-qc/9505028.

[2] L. Smolin, A holograpic formulation of quantum general relativity, Phys. Rev. D61 (2000) 084007, hep-th/9808191.

[3] Y. Ling and L. Smolin, The supersymmetric spin networks and quantum supergravity, Phys. Rev. D61, 044008(2000), hep-th/9904016.

[4] Y. Ling and L. Smolin, $O s p(2 \mid 2)$ spin networks and N=2 supergravity, preprint in preparation.

[5] Y. Ling and L. Smolin, Holography, BPS states and N=2 quantum supergravity, preprint in preparation.

[6] C. Rovelli and L. Smolin Discreteness of area and volume in quantum gravity, Nuclear Physics B 442 (1995) 593. Erratum: Nucl. Phys. B 456 (1995) 734.

[7] C. Rovelli and L. Smolin, Spin networks and quantum gravity, gr-qc/9505006, Physical Review D 52 (1995) 5743-5759.

[8] L. Smolin: in Quantum Gravity and Cosmology, eds J Pérez-Mercader et al, World Scientific, Singapore 1992; The future of spin networks gr-qc/9702030 in the Penrose Feshscrift.

[9] C. Rovelli and L. Smolin, Knot theory and quantum theory, Phys. Rev. Lett 61(1988)1155; Loop representation of quantum general relativity, Nucl. Phys. B331(1990)80-152.

[10] C. Rovelli and L. Smolin, The physical hamiltonian in nonperturbative quantum gravity, Phys. Rev. Lett.72(1994)446; Spin Networks and Quantum Gravity, Phys. Rev. D52(1995)5743-5759.

[11] T. Jacobson, New Variables for canonical supergravity, Class. Quant. Grav.5(1988)923935. 
[12] D. Armand-Ugon, R. Gambini, O. Obregon, J. Pullin, Towards a loop representation for quantum canonical supergravity, hep-th/9508036, Nucl. Phys. B460 (1996) 615; L. F. Urrutia Towards a loop representation of connection theories defined over a super-lie algebra, hep-th/9609010.

[13] H. Kunitomo and T. Sano The Ashtekar formulation for canonical N=2 supergravity, Prog. Theor. Phys. suppl. (1993) 31; Takashi Sano and J. Shiraishi, The Nonperturbative Canonical Quantization of the N=1 Supergravity, Nucl. Phys. B410 (1993) 423, hep-th/9211104; The Ashtekar Formalism and WKB Wave Functions of N=1,2 Supergravities, hep-th/9211103; T. Kadoyoshi and S. Nojiri, $N=3$ and $N=4$ two form supergravities, Mod. Phys. Lett. A12:1165-1174,1997, hep-th/9703149.

[14] K. Ezawa, Ashtekar's formulation for $N=1, N=2$ supergravities as constrained BF theories, Prog. Theor. Phys.95:863-882, 1996, hep-th/9511047.

[15] L. Smolin, The Bekenstein Bound, Topological Quantum Field Theory and Pluralistic Quantum Field Theory, gr-qc/9508064.

[16] G.'Hooft, Dimensional reduction in quantum gravity, gr-qc/9310006.

[17] L. Susskind, The world as a hologram, J. Math. Phys. 36(1995)6377-6396, hepth/9409089.

[18] J. D. Bekenstein, Lett. Nuovo. Cim 11(1974).

[19] H. Kodama, Prog. Theor. Phys. 80, 1024(1988).

[20] H. Kodama, Phys. Rev. D42(1990)2548.

[21] L. Smolin and C. Soo, The Chern-Simons Invariant as the Natural Time Variable for Classical and Quantum Cosmology, Nucl. Phys. B449 (1995) 289, gr-qc/9405015.

[22] C. Rovelli, Loop Quantum Gravity, Review paper written for the electronic journal 'Living Reviews', gr-qc/9710008; L. Smolin, The future of spin networks, in the Penrose Feshscrift, gr-qc/9702030. 
[23] J. Barrett and L. Crane, Relativistic spin networks and quantum gravity, J. Math. Phys. 39 (1998) 3296-3302, gr-qc/9709028.

[24] J. Baez, Spin foam models, Class. Quant. Grav. 15 (1998) 1827-1858, gr-qc/9709052.

[25] S. Major and L. Smolin, Quantum deformation of quantum gravity, Nucl. Phys. B473, 267(1996), gr-qc/9512020; R. Borissov, S. Major and L. Smolin, The geometry of quantum spin networks, Class. and Quant. Grav.12, 3183(1996), gr-qc/9512043.

[26] R. Capovilla, J. Dell and T. Jacobson, Phys. Rev. Lett. 21, 2325(1989); Class. Quant. Grav. 8, 59(1991); R. Capovilla, J. Dell, T. Jacobson and L. Mason, Class. and Quant. Grav. 8, 41(1991).

[27] Kunish P P and Reshetikhin, N Yu, 1989, Lett. Math. Phys.18, 143; Saluer H, 1990 Nucl. Phys. B 336, 363.

[28] Y. Ling and L. Smolin, Eleven dimensional supergravity as a constrained topological field theory, hep-th/0003285.

[29] A. Ashtekar, J. Baez, K. Krasnov, Quantum Geometry of Isolated Horizons and Black Hole Entropy gr-qc/0005126; A. Ashtekar, J. Baez, A. Corichi, K. Krasnov, Quantum Geometry and Black Hole Entropy,

[30] K. Krasnov, On Quantum Statistical Mechanics of a Schwarzschild Black Hole, grqc/9605047, Gen. Rel. Grav. 30 (1998) 53-68

[31] M, Varadarajan, unpublished, Seth A. Major, Quasilocal Energy for Spin-net Gravity, gr-qc/9906052, Class.Quant.Grav. 17 (2000) 1467-1487; T. Thiemann, Quantum Poincaré Algebra and a Quantum Positivity of Energy Theorem for Canonical Quantum Gravity, gr-qc/9705020, Class.Quant.Grav. 15 (1998) 1463-1485.

[32] L. Crane in, Categorical Physics, hep-th/9301061; Topological Field theory as the key to quantum gravity, hep-th/9308126, in Knot theory and quantum gravity ed. J. Baez, (Oxford University Press); Clocks and Categories, is quantum gravity algebraic? J. Math. Phys. 36 (1995) 6180-6193, gr-qc/9504038. 
[33] F. Markopoulou and L. Smolin, Holography in a quantum spacetime, hep-th/9910146.

[34] L. Smolin, The strong and the weak holographic principles, hep-th/0003056.

[35] L. Smolin, A proposal for the background independent formulation of M theory hepth/9903166. 